\title{
A BLOCK-PARALLEL CONJUGATE GRADIENT METHOD FOR SEPARABLE QUADRATIC PROGRAMMING PROBLEMS ${ }^{1}$
}

\author{
Eiki Yamakawa \\ Takamatsu University
}

\author{
Masao Fukushima \\ Kyoto University
}

(Received November 16, 1994; Revised May 8, 1995)

\begin{abstract}
For a large-scale quadratic programming problem with separable objective function, a variant of the conjugate gradient method can effectively be applied to the dual problem. In this paper, we consider a block-parallel modification of the conjugate gradient method, which is suitable for implementation on a parallel computer. More precisely, the method proceeds in a block Jacobi manner and executes the conjugate gradient iteration to solve quadratic programming subproblems associated with respective blocks. We implement the method on a Connection Machine Model CM-5 in the Single-Program Multiple-Data model of computation. We report some numerical results, which show that the proposed method is effective particularly for problems with some block structure.
\end{abstract}

\section{Introduction}

In recent years, much effort has been made in the design of parallel algorithms for solving various optimization problems $[2,3,4,6,7,8,9,10,11,12,13,14,22,23,24,28,29]$. Many of those algorithms are of the data parallel type, which execute identical or similar operations on numerous data concurrently using many processors. The two concepts that play an important role in designing such parallel algorithms are the duality in mathematical programming and the splitting of operators or matrices involved. For example, Fukushima et al. [14] have developed a descent type algorithm for solving general convex programming problems, using a dual optimization approach incorporating the idea of operator splitting. Another useful idea from the duality theory is the alternating direction method of multipliers $[7,8,9,12]$, which is considered an ingenious dual method combined with operator splitting. On the other hand, Mangasarian and De Leone $[22,23]$ propose parallel successive over-relaxation (SOR) methods for solving the symmetric linear complementarity problem. Those methods exploit a matrix splitting that uses block diagonal parts of the matrix involved. More recently, multisplitting has turned out to be a potential idea in developing effective parallel algorithms [11, 20]. Practical large-scale problems often possess some special structure, which can be exploited in designing a variety of parallel algorithms. Zenios [28] discusses basic issues in the design and implementation of data level parallelism for problems with network structure. For problems with certain block structure, parallelizable decomposition algorithms are proposed in $[3,10]$, which may effectively be realized in the Single-Program Multiple-Data (SPMD) model of computation.

In this paper, we propose an algorithm, particularly suited for parallel computation, to

\footnotetext{
${ }^{1}$ This work was done while the first author was with ATR Human Information Processing Research Laboratories and the second author was with Nara Institute of Science and Technology.
} 
solve the following separable quadratic programming problem:

$$
\begin{array}{lll}
\mathrm{QP}: & \text { minimize } & \frac{1}{2} x^{\top} D x+c^{\top} x \\
\text { subject to } & a_{i}^{\top} x=b_{i}, \quad i \in E, \\
& a_{i}^{\top} x \leq b_{i}, \quad i \in I
\end{array}
$$

where $E$ and $I$ are finite index sets, $D$ is an $n \times n$ positive diagonal matrix, $c$ and $a_{i}$ are vectors in $R^{n}, b_{i}$ are real numbers and the superscript $T$ denotes transposition. Note that, if problem QP is feasible, it has a unique solution because of the strong convexity of the objective function. Quadratic programming problems with a separable objective function arise in various network flow problems [28].

Let $A$ and $b$ denote the matrix whose $i$-th row is $a_{i}^{\top}, i \in E \cup I$, and the vector whose $i$-th element is $b_{i}, i \in E \cup I$, respectively. The dual of problem QP can be defined as

$$
\text { DQP : } \begin{array}{lc}
\text { minimize } & \frac{1}{2} z^{\top} M z+q^{\top} z \\
& \text { subject to } \\
& z_{i} \geq 0, \quad i \in I,
\end{array}
$$

where

$$
\begin{aligned}
M & =A D^{-1} A^{\top} \\
q & =A D^{-1} c+b .
\end{aligned}
$$

From the standard duality theory [21], we can easily verify that the optimal solution $x^{*}$ of problem QP is related to an optimal solution $z^{*}$ of problem DQP by

$$
x^{*}=-D^{-1}\left(A^{\top} z^{*}+c\right) .
$$

Let $r$ denote the negative gradient of the dual objective function at $z$, that is,

$$
r=-M z-q
$$

Then the Karush-Kuhn-Tucker conditions for problem DQP may be written as

$$
\begin{array}{ll}
r_{i}=0, & i \in E, \\
r_{i} \leq 0, \quad z_{i} \geq 0, \quad r_{i} z_{i}=0, & i \in I .
\end{array}
$$

Since $M$ is positive semidefinite, conditions (1.3) are not only necessary but also sufficient for optimality of problem DQP. Finding a $z$ satisfying (1.3) is in general a mixed linear complementarity problem. In particular, when the index set $E$ is empty, (1.3) becomes the symmetric linear complementarity problem

$$
M z+q \geq 0, \quad z \geq 0, \quad z^{\top}(M z+q)=0
$$

while, in the case where the index set $I$ is empty, (1.3) reduces to the system of linear equations

$$
M z+q=0 .
$$

The algorithm proposed in this paper is a block-parallel modification of the conjugate gradient method applied to the mixed linear complementarity problem (1.3). More precisely, the algorithm exploits a splitting of matrix $M$ such that $M=G+H$ with $G$ being a block diagonal matrix. Thus the algorithm may be regarded as a block Jacobi method. 
The resulting subproblems involving matrix $G$ are decomposed into small mixed linear complementarity problems, which can be solved independently from each other. Those mixed linear complementarity problems are solved using a conjugate gradient method, which is a modification of the one given in [16] and is extended to deal with simple bounds. This approach is particularly effective when the problem is sparse, because it only requires simple operations on the vectors $a_{i}$. In the special case where the block diagonal matrix $G$ is actually a diagonal matrix, the proposed algorithm reduces to the classical point Jacobi method. Although the point Jacobi method is suited for massively parallel computations, but often suffers slow convergence because of small steps. The block Jacobi modification usually leads to larger steps and hence accelerated convergence is expected. To examine the practical efficiency of the proposed algorithm, it is implemented on a Connection Machine Model CM-5 in the SPMD model of computation. Particular attention will be paid on the efficiency of the algorithm on problems with block angular structure.

This paper is organized as follows. A splitting method for problem DQP is presented and its convergence properties are discussed in Section 2. A block-parallel algorithm for problem QP is then developed in Section 3. Implementation strategies for the proposed algorithm in the SPMD model of computation are described in Section 4. Some computational results on the Connection Machine Model CM-5 are reported in Section 5. Conclusions are given in Section 6.

Throughout the paper we shall adopt the following notation. Let $J$ be an index set. For a vector $u, u_{J}$ denotes the subvector of $u$ with components $u_{i}, i \in J$. For matrix $A, A_{J}$ denotes the submatrix of $A$ consisting of the rows $a_{i}^{\top}, i \in J$.

\section{Basic Splitting Method}

In this section, we describe a splitting method for solving problem DQP, which is a natural modification of that for the symmetric linear complementarity problem [5]. A pair $(G, H)$ of matrices is called a splitting of matrix $M$ if

$$
M=G+H .
$$

A splitting $(G, H)$ is said to be regular if $G-H$ is positive definite [5, p. 400]. Since $M$ is positive semi-definite (see (1.1)), $G=(M+G-H) / 2$ is positive definite for any regular splitting $(G, H)$.

Using a regular splitting $(G, H)$ of $M$, a splitting method for the mixed linear complementarity problem (1.3) generates a sequence $\left\{z^{(k)}\right\}$ by the following iterative process.

\section{Splitting Method (Prototype) :}

Given $z^{(k)}$, find a solution $z^{(k+1)}$ of the mixed linear complementarity problem

$$
\begin{array}{ll}
\left(G z+v^{(k)}\right)_{i}=0, & i \in E \\
\left(G z+v^{(k)}\right)_{i} \geq 0, \quad z_{i} \geq 0, \quad\left(G z+v^{(k)}\right)_{i} z_{i}=0, & i \in I
\end{array}
$$

where

$$
v^{(k)}=H z^{(k)}+q
$$

If $G$ is symmetric, then subproblem (2.2) is equivalent to the following quadratic programming problem:

$$
\begin{array}{ccc}
\operatorname{DSP}^{(k)}: & \begin{array}{l}
\text { minimize } \\
\text { subject to }
\end{array} & \frac{1}{2} z^{\top} G z+\left(v^{(k)}\right)^{\top} z \\
z_{i} \geq 0,
\end{array} \quad i \in I .
$$


Since $G$ is positive definite as noted above, subproblem $\operatorname{DSP}^{(k)}$ has a unique solution for each $k$.

General convergence results of the splitting method for symmetric linear complementarity problems have recently been obtained by Luo and Tseng [18] and Iusem [17]. Moreover, Luo and Tseng [19] have established the convergence of the splitting method for symmetric affine variational inequality problems, which contain as special cases various optimization problems. In particular, the mixed linear complementarity problem (1.3) can be represented as the affine variational inequality problem of finding a $z^{*} \in Z$ such that

$$
\left(z-z^{*}\right)^{\top}\left(M z^{*}+q\right) \geq 0, \quad{ }^{\forall} z \in Z,
$$

where $Z$ is the polyhedral convex set in $R^{|E|+|I|}$ defined by

$$
Z=\left\{z \mid z_{i} \in R, i \in E, \quad \text { and } \quad z_{i} \geq 0, i \in I\right\} .
$$

Moreover, the solution $z^{(k+1)}$ of the mixed linear complementarity problem (2.2) is the vector in $Z$ that satisfies the variational inequality

$$
\left(z-z^{(k+1)}\right)^{\top}\left(G z^{(k+1)}+v^{(k)}\right) \geq 0, \quad{ }^{\forall} z \in Z .
$$

Therefore, the convergence of the splitting method for solving the mixed linear complementarity problem (1.3) can be deduced from the general results established in [19].

Theorem 1. If problem $\mathrm{QP}$ is feasible, then the sequence $\left\{z^{(k)}\right\}$ generated by the splitting method converges to an optimal solution of problem DQP at least linearly in the root sense.

Proof : Under our blanket assumptions, if problem QP is feasible, it has a unique solution. Then, from the standard duality theory [21], problem DQP also has a solution and hence the objective function of problem DQP is bounded from below on $Z$. Consequently the assumptions of Theorem 3.2 in [19] are satisfied and the desired results follow.

\section{Block-Parallel Algorithm}

By appropriately choosing matrix $G$ involved in subproblem $\operatorname{DSP}^{(k)}$, a block-parallel algorithm for problem QP is derived from the splitting method described in the previous section. To see this, let $J_{\ell} ; \ell=1, \cdots, L$, be index sets such that

$$
\bigcup_{\ell=1}^{L} J_{\ell}=E \cup I \quad \text { and } \quad J_{\ell} \cap J_{\ell^{\prime}}=\emptyset, \quad \ell \neq \ell^{\prime} .
$$

Suppose that we have a regular splitting $(G, H)$ of matrix $M$ such that, by reordering the rows and the columns if necessary, $G$ is a block diagonal matrix of the form

$$
G=\left(\begin{array}{cccc}
G_{1} & 0 & \cdots & 0 \\
0 & G_{2} & \ddots & \vdots \\
\vdots & \ddots & \ddots & 0 \\
0 & \cdots & 0 & G_{L}
\end{array}\right)
$$

where $G_{\ell}$ are $\left|J_{\ell}\right| \times\left|J_{\ell}\right|$ symmetric matrices whose rows and columns correspond to the indices in $J_{\ell}$. Then, subproblem DSP ${ }^{(k)}$ can be decomposed into $L$ independent subproblems $\operatorname{DSP}_{\ell}^{(k)}$, $\ell=1, \cdots, L$, as follows:

$$
\operatorname{DSP}_{\ell}^{(k)}: \begin{aligned}
& \text { minimize } \\
& \text { subject to }
\end{aligned} \begin{gathered}
\frac{1}{2} z_{J_{\ell}}^{\top} G_{\ell} z_{J_{\ell}}+\left(v_{J_{\ell}}^{(k)}\right)^{\top} z_{J_{\ell}} \\
z_{i} \geq 0,
\end{gathered} \quad i \in I \cap J_{\ell},
$$


where $v_{J_{\ell}}^{(k)}$ is the subvector of $v^{(k)}$ given by (2.3). Since $G$ is positive definite, matrices $G_{\ell}$ are also positive definite. Thus, each subproblem $\operatorname{DSP}_{\ell}^{(k)}$ always has a unique solution.

Note that the vector $v_{J_{\ell}}^{(k)}$ involved in each subproblem $\mathrm{DSP}_{\ell}^{(k)}$ is calculated in the following way. For the current dual estimate $z^{(k)}$, let

$$
x^{(k)}:=-D^{-1}\left(A^{\top} z^{(k)}+c\right) .
$$

Then, $x^{(k)}$ can be regarded as an estimate of the optimal solution of problem QP (see (1.2)). The residual vector $r^{(k)}$ at $x^{(k)}$ for the constraints of problem QP is defined by

$$
r^{(k)}:=A x^{(k)}-b .
$$

Since (3.1) and (3.2) together with (1.1) imply

$$
r^{(k)}=-M z^{(k)}-q,
$$

it follows from (2.1) that

$$
\begin{aligned}
v^{(k)} & :=H z^{(k)}+q \\
& =(M-G) z^{(k)}+q \\
& =-G z^{(k)}-r^{(k)} .
\end{aligned}
$$

Since $G$ is block diagonal, we have

$$
v_{J_{\ell}}^{(k)}=-G_{\ell} z_{J_{\ell}}^{(k)}-r_{J_{\ell}}^{(k)}, \quad \ell=1, \cdots, L
$$

which means that each subproblem $\operatorname{DSP}_{\ell}^{(k)}$ is completely defined using the data available within block $\ell$.

Let $I^{(k)}$ denote the index set of active constrains of problem DQP at $z^{(k)}$ such that

$$
I^{(k)}:=\left\{i \in I \mid z_{i}^{(k)}=0 \text { and } r_{i}^{(k)} \leq 0\right\} .
$$

Then, it is easy to see that $z^{(k)}$ and $r^{(k)}$ satisfy conditions (1.3) if and only if

$$
r_{i}^{(k)}=0, \quad i \in E \cup \bar{I}^{(k)}
$$

where

$$
\bar{I}^{(k)}=I \backslash I^{(k)} .
$$

Thus, we may terminate the iteration of the splitting method when conditions (3.4) hold.

To summarize, we obtain the following block-parallel algorithm for problem QP.

\section{Algorithm BP :}

Step 1. Choose an initial dual estimate $z^{(1)}$ with components $z_{i}^{(1)} \in R, i \in E$, and $z_{i}^{(1)} \geq 0$, $i \in I$. Set $k:=1$.

Step 2. Compute

$$
\begin{aligned}
x^{(k)} & :=-D^{-1}\left(A^{\top} z^{(k)}+c\right) \\
r^{(k)} & :=A x^{(k)}-b
\end{aligned}
$$

and let

$$
\begin{aligned}
& I^{(k)}:=\left\{i \in I \mid z_{i}^{(k)}=0 \text { and } r_{i}^{(k)} \leq 0\right\} \\
& \bar{I}^{(k)}:=I \backslash I^{(k)} .
\end{aligned}
$$

If $r_{i}^{(k)}=0, i \in E \cup \bar{I}^{(k)}$, then stop. (comment: $z^{(k)}$ and $x^{(k)}$ are optimal solutions of problem DQP and problem QP, respectively.) 
Step 3. For $\ell=1, \cdots, L$, find the solutions $z_{J_{\ell}}^{(k+1)}$ of subproblems $\operatorname{DSP}_{\ell}^{(k)}$.

Step 4. Set $k:=k+1$ and go to Step 2 .

Step 3 of Algorithm BP can be executed in parallel for $J_{\ell}, \ell=1, \cdots, L$. We solve each subproblem $\operatorname{DSP}_{\ell}^{(k)}$ by applying a slight modification of the conjugate gradient (CG) method [16], which uses an active set strategy to deal with simple bounds.

Let the objective function of subproblem $\operatorname{DSP}_{\ell}^{(k)}$ be denoted by

$$
\psi_{\ell}^{(k)}\left(z_{J_{\ell}}\right)=\frac{1}{2} z_{J_{\ell}}^{\top} G_{\ell} z_{J_{\ell}}+\left(v_{J_{\ell}}^{(k)}\right)^{\top} z_{J_{\ell}} \cdot
$$

The steepest descent direction of $\psi_{\ell}^{(k)}$ at $z_{J_{\ell}}^{(k)}$ is then calculated as

$$
\begin{aligned}
-\nabla \psi_{\ell}^{(k)}\left(z_{J_{\ell}}^{(k)}\right) & =-G_{\ell} z_{J_{\ell}}^{(k)}-v_{J_{\ell}}^{(k)} \\
& =r_{J_{\ell}}^{(k)}
\end{aligned}
$$

where the last equality follows from (3.3). Thus, we can execute the CG iterations using $z_{J_{\ell}}^{(k)}$ and $r_{J_{\ell}}^{(k)}$ as an initial estimate of a solution and an initial search direction, respectively. In particular, there is no need to calculate the vector $v_{J}^{(k)}$ explicitly.

To improve the speed of convergence, we precondition $G_{\ell}$ as

$$
\hat{G}_{\ell}=W_{\ell} G_{\ell} W_{\ell}^{\top}
$$

where $W_{\ell}$ is a nonsingular matrix chosen so that the condition number of $\hat{G}_{\ell}$ is smaller than that of $G_{\ell}[15, \S 9.2]$. Assuming that the diagonal elements of $G$ are all positive, we adopt here the simple preconditioning called diagonal scaling that uses

$$
W_{\ell}=\operatorname{diag}\left\{\frac{1}{\sqrt{g_{i i}}}\right\}_{i \in \mathrm{J}_{\ell}},
$$

where $g_{i i}$ are the diagonal elements of $G$. Then, since $W_{\ell}$ is positive and diagonal, subproblem $\operatorname{DSP}_{\ell}^{(k)}$ is equivalent to the problem

$$
\begin{array}{lc}
\operatorname{minimize} & \frac{1}{2} \hat{z}_{J_{\ell}}^{\top} \hat{G}_{\ell} \hat{z}_{J_{\ell}}+\left(\hat{v}_{J_{\ell}}^{(k)}\right)^{\top} \hat{z}_{J_{\ell}} \\
\text { subject to } & \hat{z}_{i} \geq 0,
\end{array} \quad i \in I \cap J_{\ell},
$$

where $\hat{z}_{J_{\ell}}=W_{\ell}^{-1} z_{J_{\ell}}$ and $\hat{v}_{J_{\ell}}^{(k)}=W_{\ell} v_{J_{\ell}}^{(k)}$. The preconditioned conjugate gradient (PCG) method stated below is in essence a CG method applied to this problem. Now let $\hat{\psi}_{\ell}^{(k)}$ denote the objective function of the latter problem. Then, since $\nabla \hat{\psi}_{\ell}^{(k)}\left(\hat{z}_{J_{\ell}}\right)=W_{\ell} \nabla \psi_{\ell}^{(k)}\left(z_{J_{\ell}}\right)$, the initial search direction $r_{J_{\ell}}^{(k)}$ is in particular modified as $Y_{\ell} r_{J_{\ell}}^{(k)}$, where $Y_{\ell}=W_{\ell}^{2}=$ $\operatorname{diag}\left\{1 / g_{i i}\right\}_{i \in J_{\ell}}$.

Now we state the preconditioned CG procedure to find a solution of subproblem $\operatorname{DSP}_{\ell}^{(k)}$. 


\section{Procedure PCG :}

Step 1. Let $Y_{\ell}\left(=\operatorname{diag}\left\{y_{i}\right\}_{i \in J_{\ell}}\right):=\operatorname{diag}\left\{1 / g_{i i}\right\}_{i \in J_{\ell}}$. Set

$$
\begin{aligned}
z_{J_{\ell}} & :=z_{J_{\ell}}^{(k)}, \\
r_{J_{\ell}} & :=r_{J_{\ell}}^{(k)}, \\
E_{\ell} & :=E \cap J_{\ell} .
\end{aligned}
$$

Step 2. Set

$$
\begin{aligned}
I_{\ell} & :=\left\{i \in I \cap J_{\ell} \mid z_{i}=0 \quad \text { and } \quad r_{i} \leq 0\right\}, \\
\bar{I}_{\ell} & :=\left(I \cap J_{\ell}\right) \backslash I_{\ell} .
\end{aligned}
$$

If $r_{i}=0, i \in E_{\ell} \cup \bar{I}_{\ell}$, then stop. (comment: $z_{J_{\ell}}$ is an optimal solution of subproblem $\operatorname{DSP}_{\ell}^{(k)}$.)

Step 3. Let $p_{J_{\ell}}$ be the vector such that

$$
p_{i}:= \begin{cases}0, & i \in I_{\ell} \\ y_{i} r_{i}, & i \in E_{\ell} \cup \bar{I}_{\ell} .\end{cases}
$$

Step 4. Compute

$$
\begin{aligned}
s_{J_{\ell}} & :=G_{\ell} p_{J_{\ell}}, \\
\zeta_{\ell} & :=p_{J_{\ell}}^{\top} s_{J_{\ell}}, \\
\xi_{\ell} & :=p_{J_{\ell}}^{\top} r_{J_{\ell}}, \\
\alpha_{\ell} & :=\xi_{\ell} / \zeta_{\ell} .
\end{aligned}
$$

If $z_{J_{\ell}}+\alpha_{\ell} p_{J_{\ell}} \geq 0$, then go to Step 5. Otherwise, go to Step 6 .

Step 5. Set

$$
\begin{aligned}
& z_{J_{\ell}}:=z_{J_{\ell}}+\alpha_{\ell} p_{J_{\ell}}, \\
& r_{J_{\ell}}:=r_{J_{\ell}}-\alpha_{\ell} s_{J_{\ell}}
\end{aligned} .
$$

If $r_{i}=0, i \in E_{\ell} \cup \bar{I}_{\ell}$, then go to Step 2. Otherwise, let

$$
\begin{aligned}
\beta_{\ell} & :=\frac{1}{\xi_{\ell}} \sum_{i \in E_{\ell} \cup \bar{I}_{\ell}} y_{i} r_{i}^{2}, \\
p_{i} & := \begin{cases}0, & i \in I_{\ell} \\
y_{i} r_{i}+\beta_{\ell} p_{i}, & i \in E_{\ell} \cup \bar{I}_{\ell}\end{cases}
\end{aligned}
$$

and go to Step 4 .

Step 6. Calculate

Set

$$
\bar{\alpha}_{\ell}:=\min \left\{\frac{-z_{i}}{p_{i}} \mid p_{i}<0, i \in \bar{I}_{\ell}\right\} .
$$

$$
\begin{aligned}
z_{J_{\ell}} & :=z_{J_{\ell}}+\bar{\alpha}_{\ell} p_{J_{\ell}}, \\
r_{J_{\ell}} & :=r_{J_{\ell}}-\bar{\alpha}_{\ell} s_{J_{\ell}} \\
I_{\ell} & :=\left\{i \in I \cap J_{\ell} \mid z_{i}=0\right\} \\
\bar{I}_{\ell} & :=\left(I \cap J_{\ell}\right) \backslash I_{\ell} .
\end{aligned}
$$

If $r_{i}=0, i \in E_{\ell} \cup \bar{I}_{\ell}$, then go to Step 2. Otherwise, go to Step 3 . 
In the remainder of this section, some examples of splitting $(G, H)$ are described. The simplest choice is to let $G_{\ell}$ be a scalar multiple of the block diagonal part of matrix $M$, that is,

$$
G_{\ell}:=\omega^{-1} M_{\ell}
$$

where $\omega>0$ is a relaxation parameter and $M_{\ell}$ denotes the submatrix of $M$ consisting of the elements $a_{i}^{\top} D^{-1} a_{j},(i, j) \in J_{\ell} \times J_{\ell}$. Clearly $G$ is positive semi-definite. In addition, if the constraint matrix $A$ of problem QP has some block structure, the off-block-diagonal parts of matrix $M$ are supposed to be highly sparse. Then, a relatively large value of the relaxation parameter $\omega$ can be chosen and hence fast convergence is expected. Moreover, since each element of $G_{\ell}$ is of the form $\omega^{-1} a_{i}^{\top} D^{-1} a_{j}$, the matrix-vector multiplications involving $G_{\ell}$ in Procedure PCG can effectively be executed using only simple operations on the rows of $A$.

If matrices $M_{\ell}$ are positive definite (they are positive semi-definite by construction), then the splitting $(G, H)$ given by (3.5) is regular, provided that parameter $\omega$ is chosen sufficiently small. On the other hand, when $M_{\ell}$ are not positive definite, the splitting $(G, H)$ is not necessarily regular. In such a case, by choosing $G_{\ell}$ as

$$
G_{\ell}:=M_{\ell}+\omega^{-1} \mathcal{I}_{\ell} \quad \text { or } \quad G_{\ell}:=\omega^{-1}\left(M_{\ell}+\mathcal{I}_{\ell}\right),
$$

where $\mathcal{I}_{\ell} \in R^{\left|J_{\ell}\right| \times\left|J_{\ell}\right|}$ is the identity matrix, the splitting $(G, H)$ becomes regular for sufficiently small $\omega>0$.

The above-mentioned choices of $G$ yield sparsity-preserving CG iterations, and hence enable us to deal effectively with large-scale problems.

\section{Implementation Strategy}

Algorithm BP is of the control parallel type in the sense that multiple quadratic programming subproblems may be solved concurrently. This type of parallel algorithms can be implemented on Multiple-Instruction Multiple-Data (MIMD) systems, where individual processors run under the control of their own program. For the proposed algorithm, however, the programs in the individual processors are nearly identical because we solve each subproblem $\mathrm{DSP}_{\ell}^{(k)}$ by applying Procedure PCG. In such a case, the SPMD model of computation is convenient, in which a single program is written and all processors execute this program on their own data independently. It is also appropriate that the proposed algorithm is implemented on a distributed memory system, in which processors communicate by exchanging messages through an interconnecting network.

Now we describe the implementation strategy for the proposed algorithm. Under the multi-processor environment, one processor, which we refer to as the master node, controls major iterations, while the remaining processors called worker nodes concentrate on inner iterations. More precisely, subproblems $\mathrm{DSP}_{\ell}^{(k)}$ are solved using Procedure PCG on worker nodes in parallel. Then, making use of message passing primitives, their solutions $z_{J_{\ell}}^{(k+1)}$ are transmitted to the master node, which executes Step 2 of Algorithm BP. If conditions (3.4) are satisfied, the master node broadcasts the termination of the algorithm. Otherwise, the master node transmits the subvectors $r_{J_{\ell}}^{(k+1)}$ to the corresponding worker nodes. Then, using $r_{J_{\ell}}^{(k+1)}$ together with the previous solution $z_{J_{\ell}}^{(k+1)}$, each worker node again executes Procedure PCG to solve subproblem $\operatorname{DSP}_{\ell}^{(k+1)}$, and so forth.

The above mentioned strategy, which naturally follows from the construction of Algorithm BP, is slightly inefficient because each worker node remains idle while the master node executes Step 2 of Algorithm BP. In fact, the most time consuming part of the algorithm 
Worker Node 1

Master Node

Worker Node 2

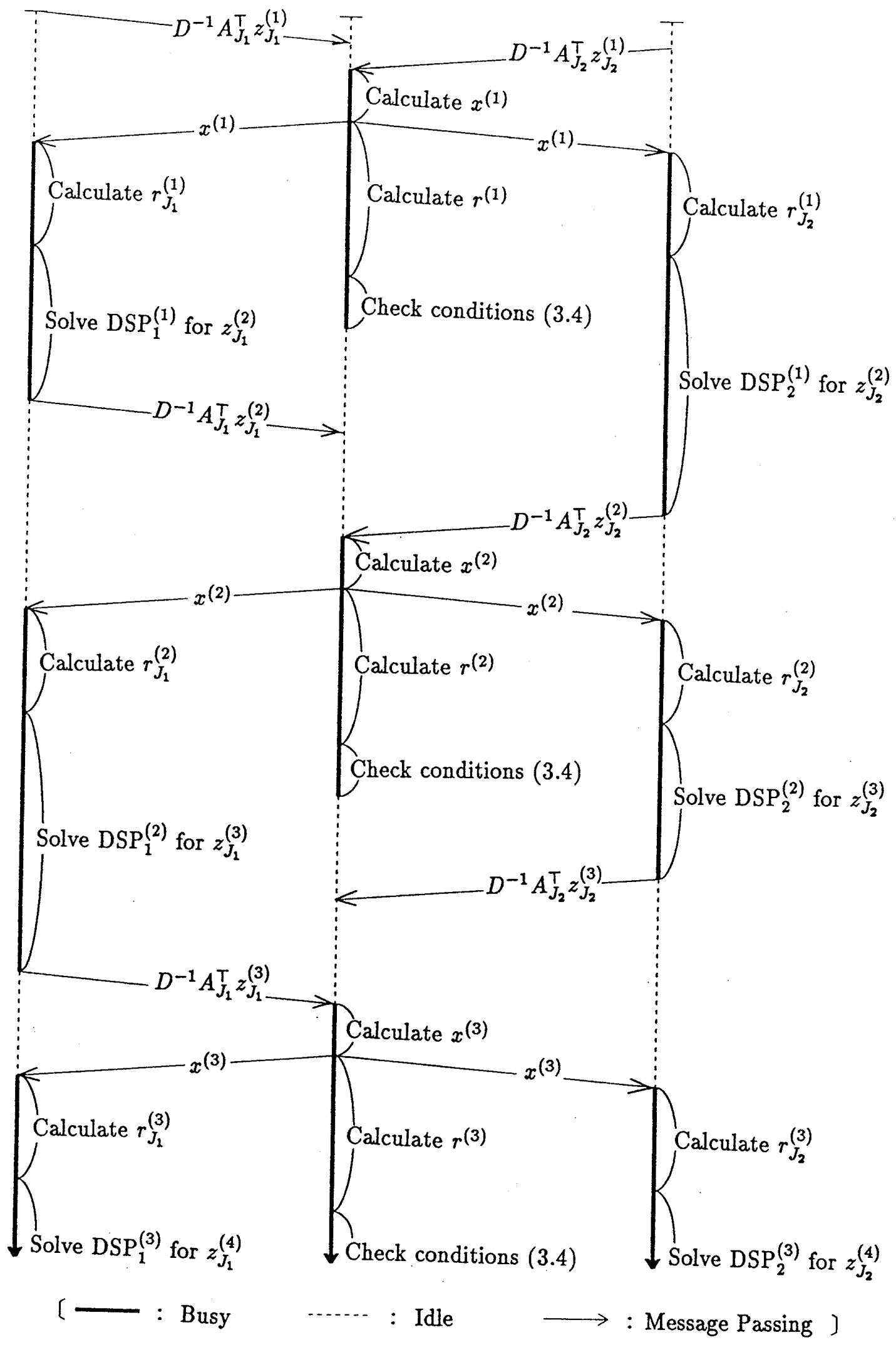

Figure 1: Execution sequence for the proposed algorithm when $L=2$. 
is the matrix-vector multiplications $A^{\top} z^{(k)}$ and $A x^{(k)}$. To reduce the idle time, we modify the implementation strategy in the following way. Since Procedure PCG consists of simple operations on the rows of submatrix $A_{J_{\ell}}$, we replace the message $z_{J_{\ell}}^{(k+1)}$ from each worker node to the master node by $D^{-1} A_{J_{\ell}}^{\top} z_{J_{\ell}}^{(k+1)}$. Then, the master node calculates an estimate $x^{(k+1)}$ of the optimal solution of problem QP simply as

$$
x^{(k+1)}=-\sum_{\ell=1}^{L} D^{-1} A_{J_{\ell}}^{\top} z_{J_{\ell}}^{(k+1)}-D^{-1} c
$$

and immediately broadcasts it to all worker nodes. Moreover, before solving subproblem $\mathrm{DSP}_{\ell}^{(k+1)}$, each worker node independently calculates the subvector of the residual $r^{(k+1)}$ as

$$
r_{J_{\ell}}^{(k+1)}=A_{J_{\ell}} x^{(k+1)}-b_{J_{\ell}}
$$

On the other hand, the residual $r^{(k+1)}$ is also calculated on the master node and the termination criterion (3.4) is checked while the worker nodes are solving subproblems $\operatorname{DSP}_{\ell}^{(k+1)}$. An example of the execution sequence for the proposed algorithm is illustrated in Figure 1.

Finally, we briefly describe how matrix $A$, which is decomposable into smaller sparse blocks $A_{J_{\ell}}$, is represented. On the master node and each worker node, all working variables used in Algorithm BP and Procedure PCG are stored in one-dimensional arrays whose lengths are equal to the number of non-zero elements of $A$ and $A_{J_{\ell}}$, respectively. Moreover, we store the non-zero elements of $a_{i}$ in a contiguous segment for each index $i \in J_{\ell}$. Then, the segmented scan operation $[29, \S 3.2]$ enables us to perform matrix-vector multiplications associated with matrix $A$ or its submatrices $A_{J_{\ell}}$ effectively on their segments. If data level parallelism is available on the master node and each worker node, these multiplications are executed, in fact, in parallel.

\section{Numerical Results}

In this section, we report some numerical results with the proposed algorithm on the Connection Machine Model CM-5 at ATR Human Information Processing Research Laboratories. The particular configuration of the CM-5 is 32 processing nodes (PN's).' Each PN has 4 vector floating point units (VFPU's) and 4 banks of 8 Mbytes local memories. The performance of each PN is estimated as 128 MFLOPS (peak) [26]. All PN's are supervised by a control processor, which loads a program, broadcasts instructions and initiates execution. The processors of the CM-5 are interconnected by two types of communication networks; the control network for global operations such as synchronization, and the data network for bulk data transfers from one processor to another. On the CM-5, the SPMD model of computation is available, in which various algorithms of control parallel type as well as those of data parallel type can effectively be implemented.

For the proposed algorithm, we coded the procedure to be executed on each PN in the data parallel language CM Fortran [25] together with the software library CMMD [27], which provides various routines for realizing control parallel primitives such as global synchronization and message passing. To operate the strategies described in the previous section, we assigned one PN to the master node, and the other PN's to worker nodes. CM Fortran is an extended version of FORTRAN 77, which is specially designed for Connection Machine systems and equipped with array-handling facilities of Fortran 90. Array operations based on Fortran 90 map naturally onto the data parallel operations. In practice, array elements treated in each PN are automatically spread across the distributed memories attached to 
the 4 VFPU's. Moreover, under the mechanism of virtual processors [29, §3.1], each VFPU operates in a serial fashion on multiple copies of data as if multiple processors operate on their own copy of data in parallel. Therefore, inner products of vectors and sparse matrixvector multiplications involved in Algorithm BP and Procedure PCG may effectively be performed on each PN. On the other hand, communication between PN's has to be coded on the program explicitly.

We tested the proposed algorithm on randomly generated sparse quadratic programming problems of the following form:

$$
\begin{array}{lc}
\operatorname{minimize} & \frac{1}{2} x^{\top} D x+c^{\top} x \\
\text { subject to } & A x \leq b,
\end{array}
$$

where $D$ is an $n \times n$ positive diagonal matrix, $c$ is a vector in $R^{n}, A$ is an $m \times n$ matrix with some block structure and $b$ is a vector in $R^{m}$. The non-zero elements of $A$ and all elements of $b, c$ and $D$ were chosen uniformly from the intervals $[-5,5],[1,10],[-100,100]$ and $[1,10]$, respectively. Note that any problem thus generated is feasible, because $b>0$ implies that the origin $x=0$ satisfies the constraints. We used two sets of test problems; one with staircase structure, the other with block angular structure. Each set consists of problems with various size. For each problem size, we generated five problem instances using different random number seeds. The results shown in the tables and figures below are the averages over the five test problems generated.

Algorithm BP combined with Procedure PCG can be applied to problem (5.1) by setting $E=\emptyset$. In Step 2 of Algorithm BP, we stop the iteration if the conditions

$$
\left|r_{i}^{(k)}\right| \leq 10^{-7}\|b\|_{\infty}
$$

or

$$
z_{i}^{(k)}=0, \quad r_{i}^{(k)} \leq 10^{-7}\|b\|_{\infty}
$$

hold for all $i \in\{1, \cdots, m\}$. On the other hand, we terminate Procedure PCG for solving subproblem $\operatorname{DSP}_{\ell}^{(k)}$ when the current iterate satisfies either

$$
\left|r_{i}\right| \leq \varepsilon_{\ell}^{(k)}\left\|b_{J_{\ell}}\right\|_{\infty}
$$

or

$$
z_{i}=0, \quad r_{i} \leq \varepsilon_{\ell}^{(k)}\left\|b_{J_{\ell}}\right\|_{\infty}
$$

for all $i \in J_{\ell}$, where $\varepsilon_{\ell}^{(k)}$ are sufficiently small positive numbers. It should be noted that the tolerances $\varepsilon_{\ell}^{(k)}$ have to be $10^{-7}$ or less at the final stage of the major iterations. From a practical viewpoint, however, it is often effective to truncate inner iterations using relatively loose convergence criteria when the current (major) iterate is far from the optimal solution. Thus, we introduce the following strategy:

$$
\begin{array}{ll}
\varepsilon_{\ell}^{(1)}:=\max \left\{\frac{r_{i}^{(1)}}{\left\|b_{J_{\ell}}\right\|_{\infty}} \mid i \in J_{\ell}\right\}, & \\
\varepsilon_{\ell}^{(k)}:=\max \left\{10^{-7}, \frac{\varepsilon_{\ell}^{(k-1)}}{10}\right\}, \quad k>1 .
\end{array}
$$

In practice, the termination criterion (5.2) determines a vector $z^{(k+1)}$ as an approximate solution of subproblem $\operatorname{DSP}^{(k)}$, or equivalently, the affine variational inequality (2.5). Note that 
Luo and Tseng [19] have shown that, under appropriate assumptions, the sequence $\left\{z^{(k)}\right\}$ generated by approximately solving subproblems (2.5) converges linearly to an optimal solution of the symmetric affine variational inequality problem (2.4).

Throughout the computational experiments, the initial point for Algorithm BP was always chosen as $z^{(1)}=0$, and the matrices $G_{\ell}$ involved in subproblem $\operatorname{DSP}_{\ell}^{(k)}$ were set to be the block diagonal part of matrix $M$ as in (3.5). Moreover, we assume that each worker node can be assigned to solve its own subproblem $\operatorname{DSP}_{\ell}^{(k)}$ exclusively. All computations were done in double precision arithmetic.

\subsection{Staircase constraints}

We first tested the proposed algorithm on problems of the form (5.1), in which matrix $A$ has the staircase structure

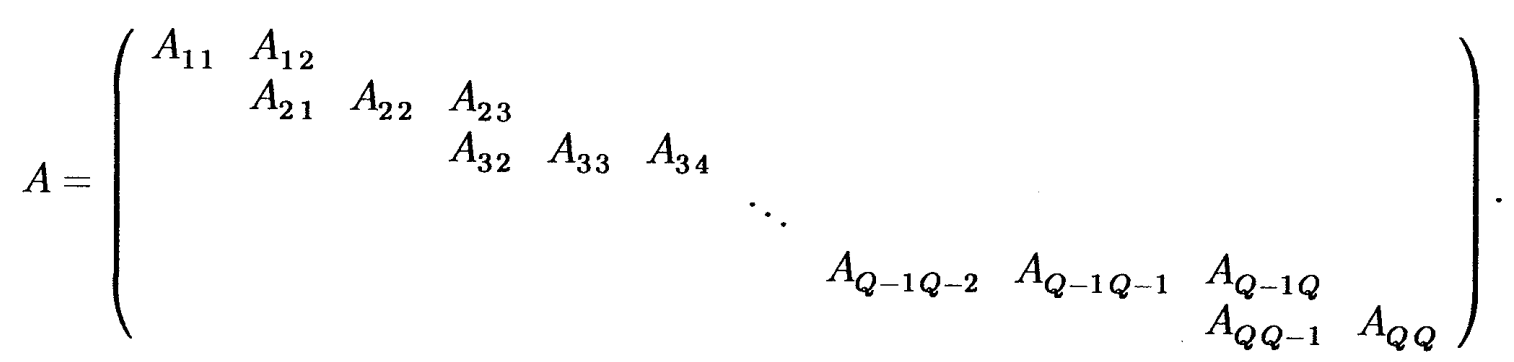

This type of problems often arise in multi-period planning and discrete-time dynamic control. Let the diagonal matrix $D$ be symmetrically partitioned to conform with the column partition of $A$, i.e.,

$$
D=\operatorname{diag}\left\{D_{11}, D_{12}, D_{22}, D_{23}, D_{33}, D_{34}, \cdots, D_{Q-1 Q-1}, D_{Q-1 Q}, D_{Q Q}\right\} .
$$

Then, matrix $M$ given by (1.1) is written as the block tri-diagonal matrix

$$
M=\left(\begin{array}{ccccc}
M_{11} & M_{12} & & & \\
M_{21} & M_{22} & M_{23} & & \\
& M_{32} & M_{33} & \ddots & \\
& & \ddots & \ddots & M_{Q-1 Q} \\
& & & M_{Q Q-1} & M_{Q Q}
\end{array}\right)
$$

where

$$
\begin{array}{rlrl}
M_{q q} & =A_{q q-1} D_{q-1 q}^{-1} A_{q q-1}^{\top}+A_{q q} D_{q q}^{-1} A_{q q}^{\top}+A_{q q+1} D_{q q+1}^{-1} A_{q q+1}^{\top}, & q=1, \cdots, Q \\
M_{q q+1}^{\top}=A_{q q+1} D_{q q+1}^{-1} A_{q+1 q}^{\top} & q=1, \cdots, Q-1,
\end{array}
$$

with $A_{10}=A_{Q Q+1}=0$. When we can use as many worker nodes as the number $Q$ of blocks, we simply determine $G_{\ell}$ using the block diagonal part $M_{\ell \ell}$ of matrix $M$. Otherwise, we set, for example,

$$
M_{\ell}=\left(\begin{array}{cccc}
M_{q_{\ell} q_{\ell}} & M_{q_{\ell} q_{\ell}+1} & 0 & 0 \\
M_{q_{\ell}+1 q_{\ell}} & M_{q_{\ell}+1 q_{\ell}+1} & M_{q_{\ell}+1 q_{\ell}+2} & 0 \\
0 & M_{q_{\ell}+2 q_{\ell}+1} & M_{q_{\ell}+2 q_{\ell}+2} & M_{q_{\ell}+2 q_{\ell}+3} \\
0 & 0 & M_{q_{\ell}+3 q_{\ell}+2} & M_{q_{\ell}+3 q_{\ell}+3}
\end{array}\right) .
$$

Since the block diagonal matrix $G$ given by (3.5) does not differ so much from the block tri-diagonal matrix $M$ of (5.3), we may expect that the proposed algorithm can effectively be applied to problems with staircase structure. 
Table 1: Characteristics of the test problems with staircase structure.

\begin{tabular}{|c|c|ccc|cc|}
\hline & \multirow{2}{*}{ Problem } & $Q$ & \multicolumn{3}{|c|}{ Each Block $^{\dagger}$} & \multicolumn{2}{c|}{ Total } \\
\cline { 3 - 7 } & $m_{q}$ & $n_{q}$ & Density of $A_{q}(\%)$ & $m$ & $n$ \\
\hline QP11 & 4 & 128 & 554 & 11.55 & 512 & 2048 \\
QP12 & 8 & 128 & 561 & 11.41 & 1024 & 4096 \\
QP13 & 16 & 128 & 564 & 11.35 & 2048 & 8192 \\
\hline QP14 & 2 & 256 & 1138 & 2.812 & 512 & 2048 \\
QP15 & 4 & 256 & 1204 & 2.658 & 1024 & 4096 \\
QP16 & 8 & 256 & 1241 & 2.579 & 2048 & 8192 \\
QP17 & 16 & 256 & 1264 & 2.538 & 4096 & 16384 \\
\hline
\end{tabular}

$\dagger$ The overlap of two consecutive blocks is about $10 \%$ in terms of the number of columns for problems QP11-QP13, and about $20 \%$ for problems QP14-QP17.

Now let $A_{q} \in R^{m_{q} \times n_{q}}$ denote the row block of matrix $A$ such that

$$
A_{q}= \begin{cases}\left(A_{11}, A_{12}\right), & q=1 \\ \left(A_{q q-1}, A_{q q}, A_{q q+1}\right), & q=2, \cdots, Q-1 \\ \left(A_{Q Q-1}, A_{Q Q}\right), & q=Q\end{cases}
$$

Then a problem with staircase structure can be characterized by the number $Q$ of blocks, the size $\left(m_{q}, n_{q}\right)$ of each block and the size $(m, n)$ of the whole matrix $A$. We generated test problems with various size, in which the ratio of $m$ to $n$ is fixed at $1 / 4$, the size of row blocks $A_{q}$ are identical and the number of non-zero elements in each matrix $A_{q}$ is fixed at 8192 . The overlap of two consecutive blocks is either about $10 \%$ or about $20 \%$ in terms of the number of columns. The characteristics of the test problems are shown in Table 1.

First of all, we made a preliminary experiment to verify the effects of the strategy based on (5.2). Test problem QP12 was used in this experiment. For comparison purposes, we also solved this test problem under the same termination criterion for inner iterations with the fixed tolerance $\varepsilon_{\ell}^{(k)}=10^{-7}$ for all $\ell$ and $k$. Table 2 shows the performance of the two strategies when the number $L$ of worker nodes is 2 and 8 . Observe that the truncation strategy (5.2) for inner iterations increases the number of major iterations very little and, more importantly, reduces the total computation time significantly.

We next examined the performance of the proposed algorithm under various choices of relaxation parameter $\omega$. In this experiment, test problems QP11-QP13 were solved using $L$ worker nodes, where $L$ varies from 2 to $Q$. The results are shown in Figure 2, in which both axes represent logarithmic scale. The symbol $\odot$ in the figure indicates the total computation

Table 2: Comparison of the truncation strategies for inner iterations.

\begin{tabular}{|c|cc|cc|}
\hline \multirow{2}{*}{$\varepsilon_{\ell}^{(k)}$} & \multicolumn{2}{|c|}{$L=2$} & \multicolumn{2}{|c|}{$L=8$} \\
\cline { 2 - 5 } & $\begin{array}{c}\text { Number of Iterations } \\
\text { Major / Inner }\end{array}$ & $\begin{array}{c}\text { CPU } \\
(\mathrm{sec})\end{array}$ & $\begin{array}{c}\text { Number of Iterations } \\
\text { Major / Inner }\end{array}$ & $\begin{array}{c}\text { CPU } \\
(\mathrm{sec})\end{array}$ \\
\hline Strategy $(5.2)$ & $19.2 / 124.0$ & 30.74 & $27.0 / 510.6$ & 13.88 \\
\hline Fixed at $10^{-7}$ & $18.6 / 569.2$ & 107.11 & $27.2 / 2349.6$ & 41.21 \\
\hline
\end{tabular}




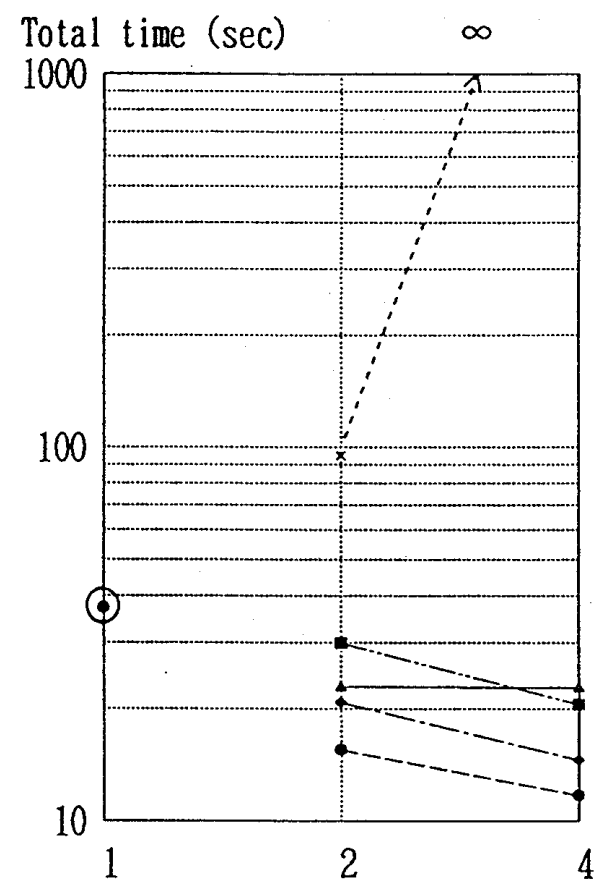

Number $L$ of worker nodes

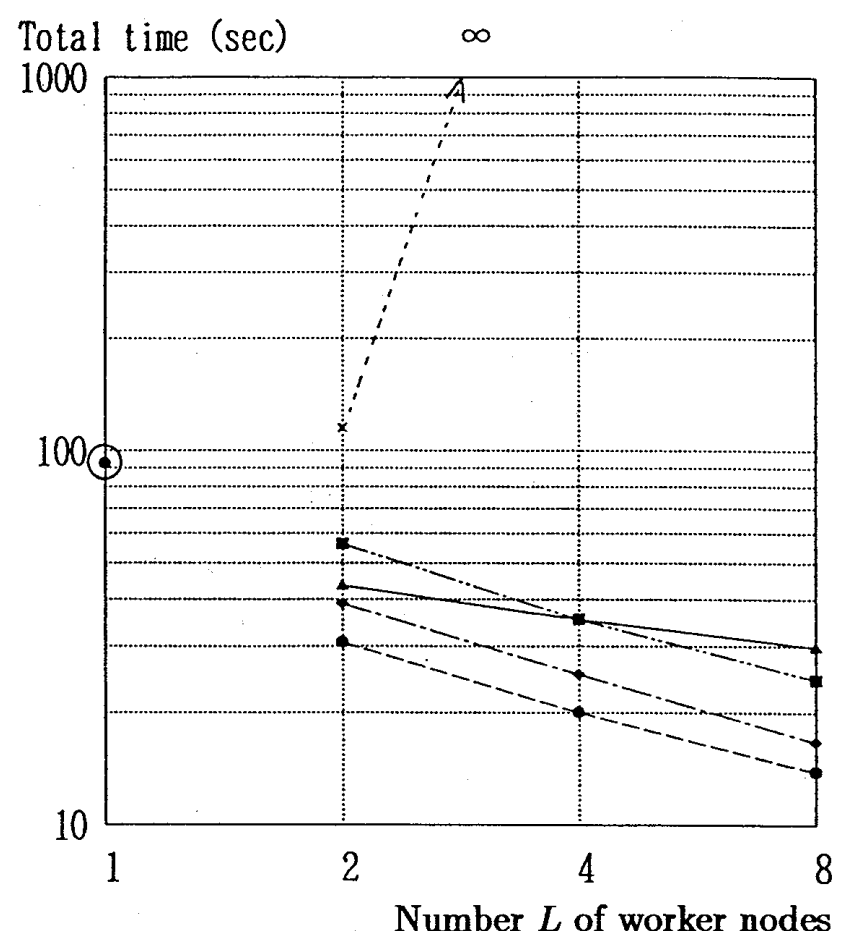

(b) Problem QP12

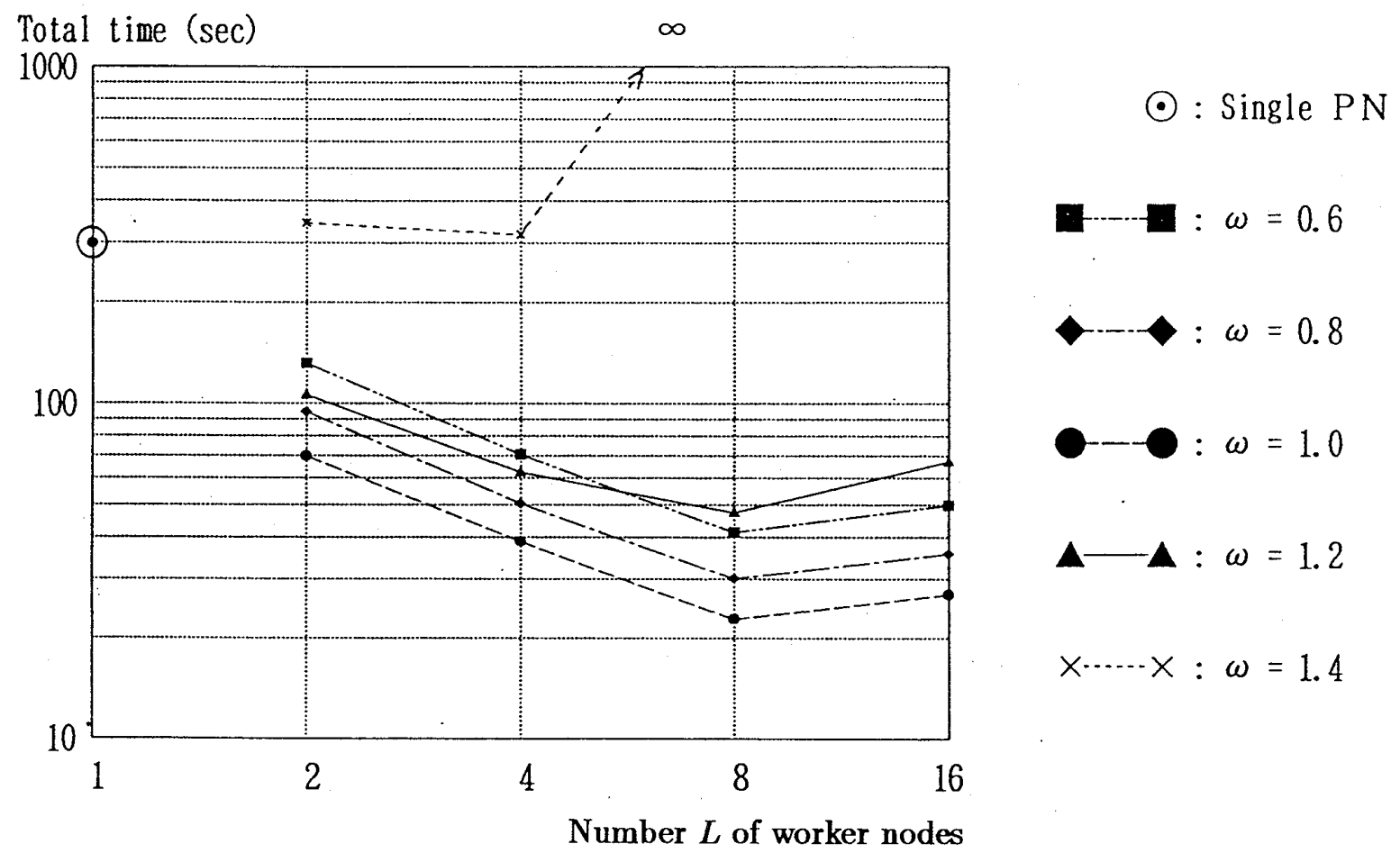

(c) Problem QP13

Figure 2: Performance of the proposed algorithm for problems with staircase structure under various choices of the relaxation parameter $\omega$. 


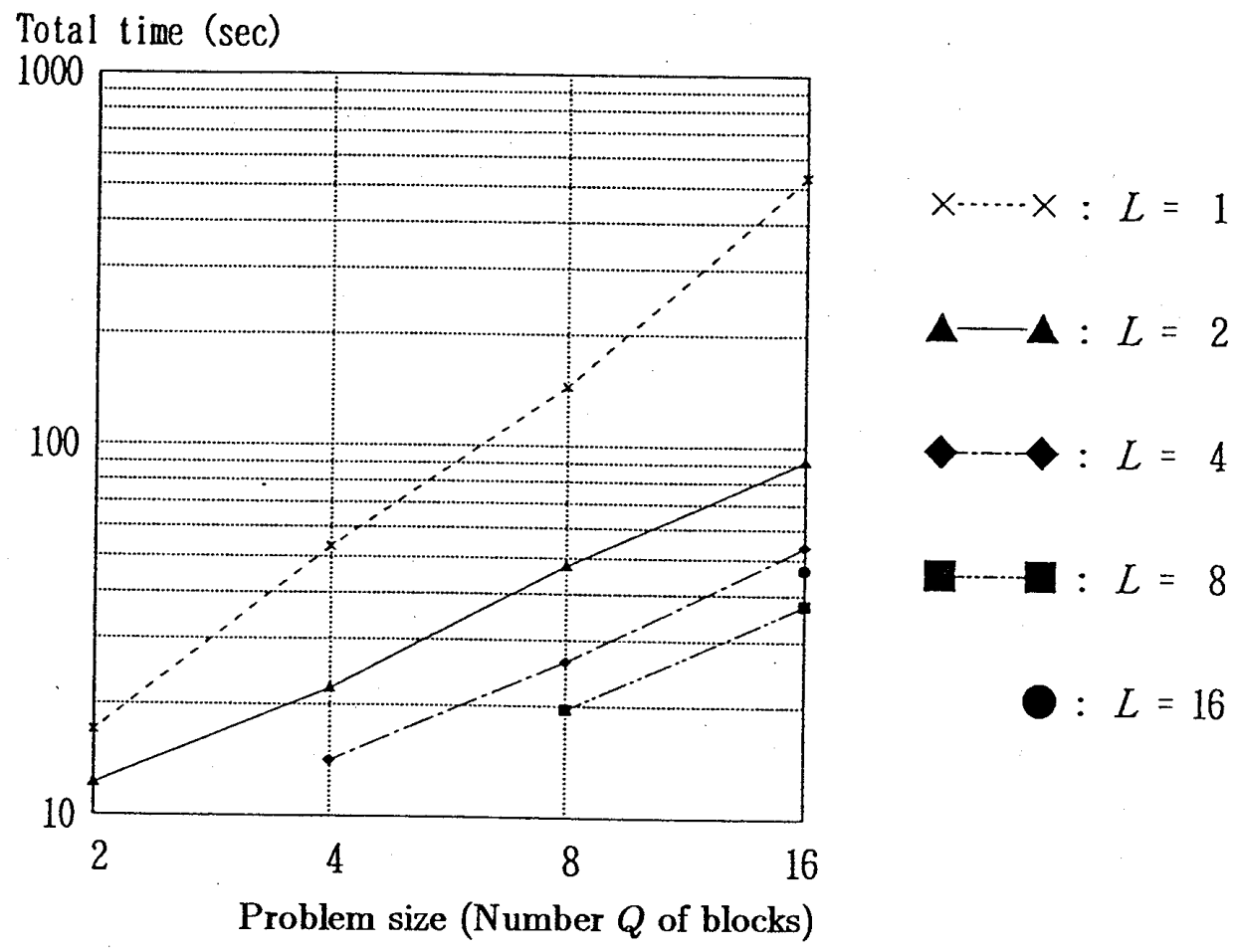

Figure 3: Performance of the proposed algorithm for problems QP14-QP17 with staircase structure.

time in the case of $L=1$ and $\omega=1.0$, which means that problem DQP is not split into subproblems and the whole problem is solved by the preconditioned conjugate gradient method (Procedure PCG) on a single PN. It is interesting to observe that the algorithm initially tends to converge faster as $\omega$ increases, but it begins to slow down when $\omega$ exceeds 1.0 , and eventually fails to converge when $\omega$ becomes 1.4 .

Then some additional experiments were made to observe the behavior of the proposed algorithm for problems with various size. We solved problems QP14-QP17 by setting relaxation parameter $\omega=1.0$. Figure 3 shows the total computation time for several choices of the number $L$ of worker nodes. Note that, for problem QP17 with 16 blocks, the case $L=16$ required more computation time than the case $L=8$. To analyze this phenomenon, we measured the details of the computation time for problem QP17. Table 3 summarizes

Table 3: Details of the computation time for problem QP17.

\begin{tabular}{|c|c|cc|c|}
\hline \multirow{2}{*}{\begin{tabular}{c} 
Number $\begin{array}{c}\text { of } \\
\text { of } \\
\text { worker nodes }\end{array}$ \\
\cline { 2 - 5 }
\end{tabular}} & Total & $\begin{array}{c}\text { Calculating } x^{(k)} \\
\text { (master) }\end{array}$ & $\begin{array}{c}\text { Busy } \\
\text { (the slowest worker) }\end{array}$ & $\begin{array}{c}\text { Communication } \\
\text { (estimate) }\end{array}$ \\
\hline 2 & 91.69 & 0.09 & 78.71 & 12.89 \\
4 & 53.81 & 0.21 & 40.62 & 12.98 \\
8 & 37.57 & 0.39 & 20.52 & 16.66 \\
16 & 46.66 & 0.78 & 12.44 & 33.44 \\
\hline
\end{tabular}


the computation time to calculate $x^{(k)}$ at the master node and the busy time at the slowest worker node, together with the communication time estimated from those data (see Figure 1). The table indicates that, when 16 worker nodes are used, the communication overhead between the master and worker nodes becomes relatively large in comparison with the computation time spent at each worker node executing Procedure PCG.

\subsection{Block angular constraints}

We also tested the proposed algorithm on problems of the form (5.1), in which matrix $A$ has the block angular structure

$$
A=\left(\begin{array}{cccc}
A_{1} & & & \\
& A_{2} & & \\
& & \ddots & \\
& & & A_{Q} \\
\bar{A}_{1} & \bar{A}_{2} & \cdots & \bar{A}_{Q}
\end{array}\right)
$$

where $A_{q}$ and $\bar{A}_{q}$ are matrices in $R^{m_{q} \times n_{q}}$ and $R^{\bar{n} \times n_{q}}$, respectively. A typical example of problems with block angular structure is a multi-commodity flow problem. Corresponding to the column partition of $A$, we may rewrite the diagonal matrix $D$ as

$$
D=\operatorname{diag}\left\{D_{1}, D_{2}, \cdots, D_{Q}\right\}
$$

where $D_{q}$ are $n_{q} \times n_{q}$ positive diagonal matrices. Then, the matrix $M$ given by (1.1) is written as follows:

where

$$
M=\left(\begin{array}{ccccc}
M_{1} & & & & \bar{M}_{1} \\
& M_{2} & & & \bar{M}_{2} \\
& & \ddots & & \vdots \\
\bar{M}_{1}^{\top} & \bar{M}_{2}^{\top} & \cdots & \bar{M}_{Q}^{\top} & M_{Q+1}
\end{array}\right)
$$

$$
\begin{array}{lll}
M_{q} & =A_{q} D_{q}^{-1} A_{q}^{\top}, & q=1, \cdots, Q, \\
\bar{M}_{q} & =A_{q} D_{q}^{-1} \bar{A}_{q}^{\dagger}, & q=1, \cdots, Q, \\
M_{Q+1} & =\sum_{q=1}^{Q} \bar{A}_{q} D_{q}^{-1} \bar{A}_{q}^{\top} . &
\end{array}
$$

When $Q+1$ worker nodes are available, (3.5) naturally suggests the simple choice of matrix $G$ such that

$$
G_{\ell}=\omega^{-1} M_{\ell}, \quad \ell=1, \cdots, L,
$$

where $L=\dot{Q}+1$. Otherwise, we set, for example,

$$
\begin{aligned}
G_{\ell}= & \omega^{-1} \operatorname{diag}\left\{M_{q_{\ell}}, M_{q_{\ell}+1}, \cdots, M_{q_{\ell+1}-1}\right\}
\end{aligned} \quad \quad \ell=1, \cdots, L-1,
$$

where $1=q_{1}<q_{2}<\cdots<q_{L} \leq Q+1$. 
Table 4: Characteristics of the test problems with block angular structure.

\begin{tabular}{|c|c|ccc|cc|}
\hline & & \multicolumn{4}{|c|}{ Each Block $^{\dagger}$} & \multicolumn{2}{c|}{ Total } \\
\cline { 3 - 7 } Problem & $Q$ & $\bar{m}$ & $\bar{n}$ & Density of $A_{q}(\%)$ & $m$ & $n$ \\
\hline QP21 & 3 & 128 & 512 & 12.5 & 896 & 1536 \\
QP22 & 7 & 128 & 512 & 12.5 & 1408 & 3584 \\
QP23 & 15 & 128 & 512 & 12.5 & 2432 & 7680 \\
\hline QP24 & 3 & 256 & 1024 & 3.125 & 1792 & 3072 \\
QP25 & 7 & 256 & 1024 & 3.125 & 2816 & 7168 \\
QP26 & 15 & 256 & 1024 & 3.125 & 4684 & 15360 \\
\hline
\end{tabular}

$\dagger$ The number of non-zero elements in $\bar{A}$ is 4096 for problems QP21 and QP24, 8192 for problems QP22 and QP25, and 16384 for problems QP23 and QP26.

Now let $\bar{A} \in R^{\bar{n} \times n}$ denote the coefficient matrix of the coupling constraints, i.e.,

$$
\bar{A}=\left(\bar{A}_{1}, \bar{A}_{2}, \cdots, \bar{A}_{Q}\right)
$$

We generated test problems with various size, in which $m_{q}=\bar{m}$ and $n_{q}=\bar{n}$ for all $q=$ $1, \cdots, Q$, the ratio of $\bar{m}$ to $\bar{n}$ is fixed at $1 / 4$ and the number of non-zero elements in each matrix $A_{q}$ is fixed at 8192. The characteristics of the test problems are shown in Table 4.

The performance of the proposed algorithm under various choices of relaxation parameter $\omega$ is examined. Figure 4 shows the results for problems QP21-QP23. The general feature of the results is similar to that for problems with staircase structure. Note that, when $\omega \geq 1.4$, the algorithm fails to solve all the test problems generated for any choice of $L$.

We then solved problems QP24-QP26 to ascertain the effectiveness of the proposed algorithm for problems with various size. In this experiment, the relaxation parameter $\omega$ is fixed at 1.0. Figure 5 shows the relationship between the computation time and the problem size measured in term of the number $Q+1$ of blocks, for several choices of the number $L$ of worker nodes.

It is well known that the concept of efficiency is useful to measure what is gained by parallel computation (see, for example, [1, p. 14] and [15, p. 65]). We define the efficiency $e_{p}$ of the proposed algorithm as follows:

$$
e_{p}:=\frac{\bar{T}}{p T_{p}},
$$

where $\bar{T}$ is the total computation time in the case of single PN, while $T_{p}$ is the total computation time using $p$ PN's. Since the algorithm is executed using $L$ worker nodes and one master node, $p$ is related to $L$ by $p=L+1$. Note that $e_{p}$ may exceed 1 , because $\bar{T}$ represents the computation time of the serial algorithm that does not utilize splitting but essentially applies Procedure PCG to problem DQP directly. Table 5 shows the efficiency of the proposed algorithm for problems QP24-QP26. It can be seen from the table that high efficiency is achieved, especially when the number of blocks is large.

\section{Conclusion}

We have presented a block-parallel modification of the conjugate gradient method for solving separable quadratic programming problems. As shown in the preceding sections, 
Total time (sec)

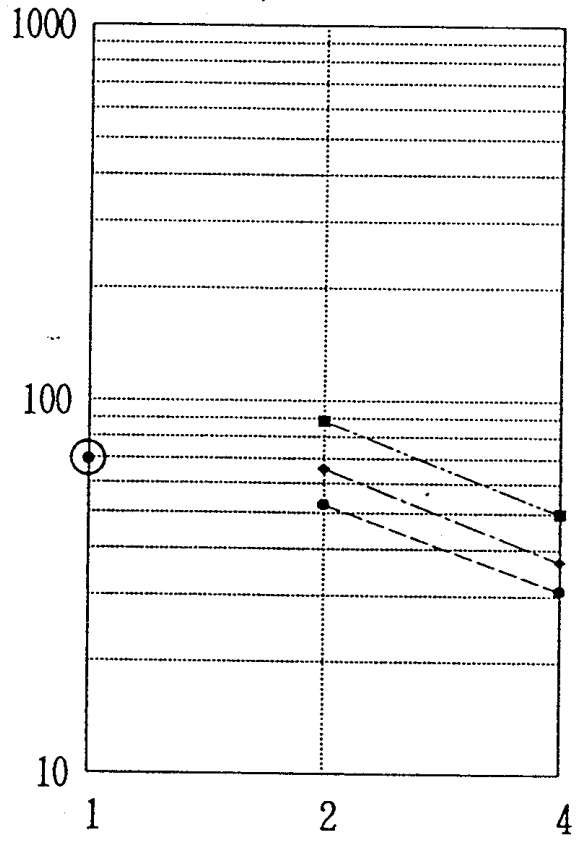

Number $L$ of worker nodes

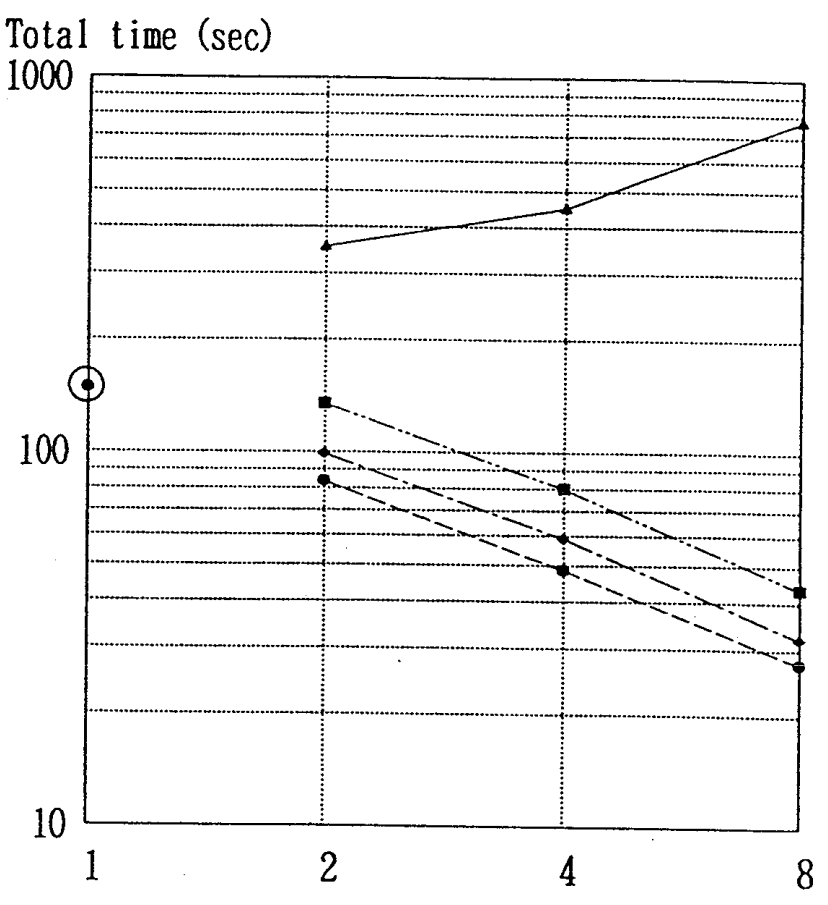

Number $L$ of worker nodes

(a) Problem QP21 ${ }^{\dagger}$

(b) Problem QP22
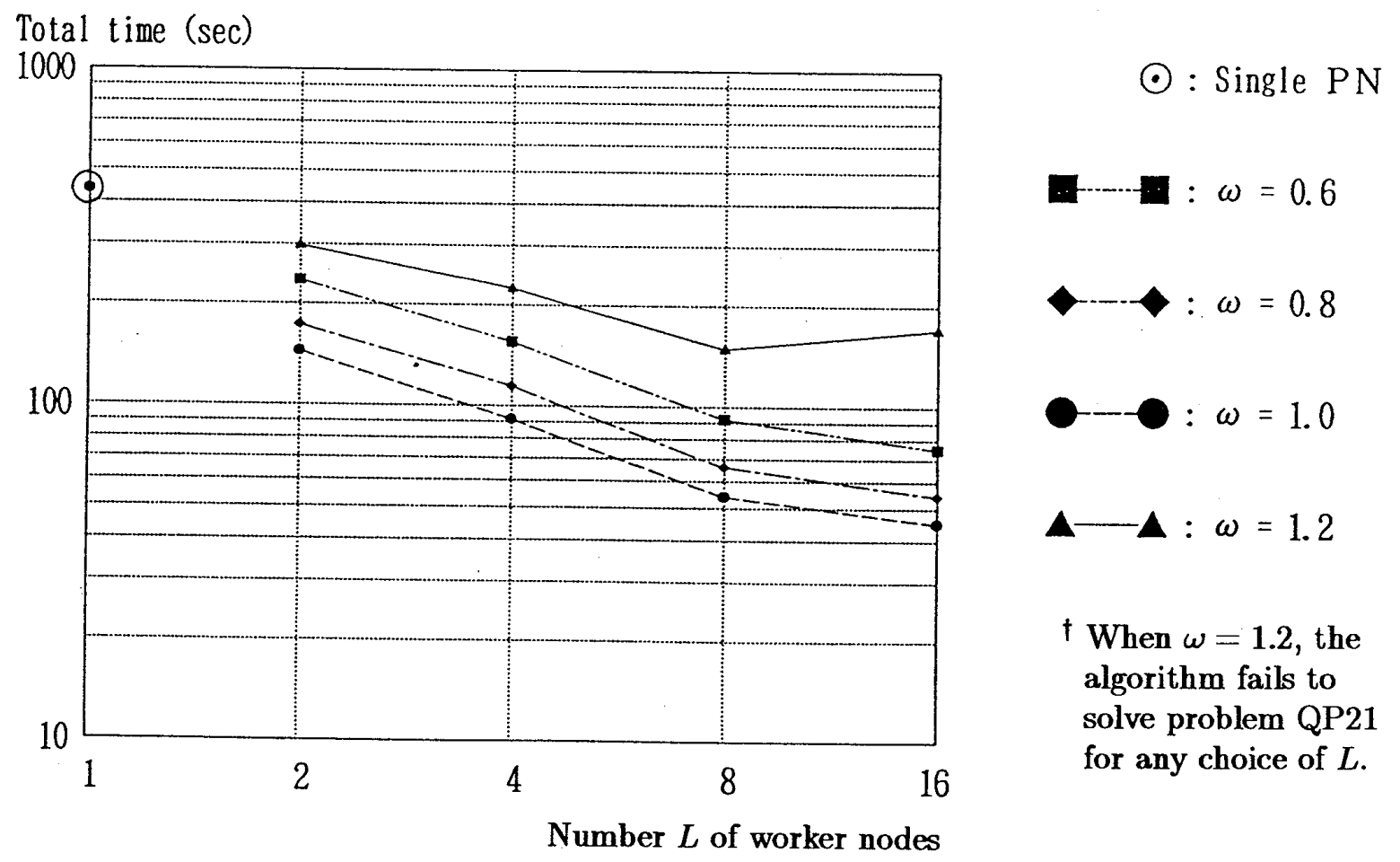

(c) Problem QP23

Figure 4: Performance of the proposed algorithm for problems with block angular structure under the various choices of the relaxation parameter $\omega$. 


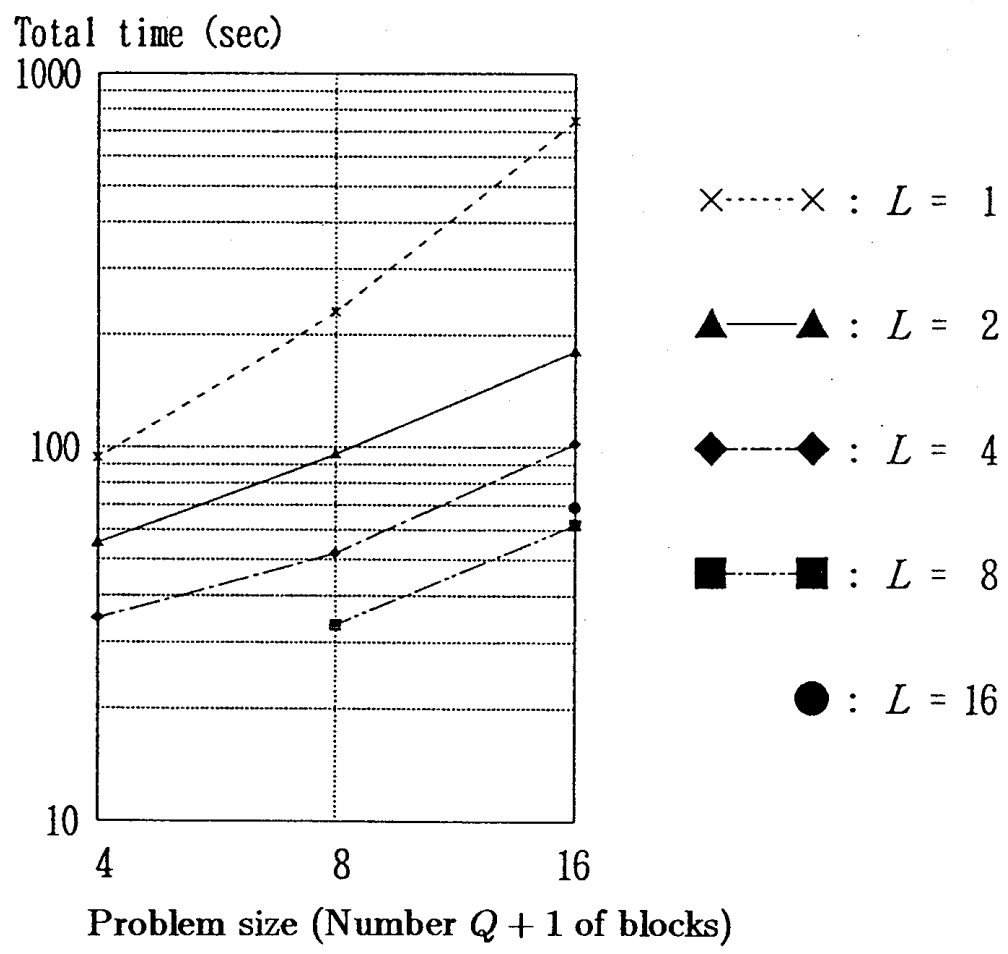

Figure 5: Performance of the proposed algorithm for problems QP24-QP26 with block angular structure.

the proposed algorithm can effectively be implemented on a parallel computer on which each processor can execute its own operations. The numerical results reported in Section 5 indicate that the present approach is particularly effective when the problems to be solved have some block structure. In our experiments, the number of worker nodes was larger than that of decomposed subproblems, so that each worker node was in charge of one subproblem only. This is not an indispensable requirement, however. It is possible to implement the algorithm by assigning more than one subproblems to each worker node (see, e.g., [3]).

The proposed algorithm is synchronous in the sense that each processor has to complete its conjugate gradient iterations before the algorithm proceeds to the next major iteration. Thus the performance of the algorithm could be seriously affected if the amounts of conjugate

Table 5: Efficiency $e_{\boldsymbol{p}}$ of the proposed algorithm. ${ }^{\dagger}$

\begin{tabular}{|c|cccc|}
\hline & \multicolumn{4}{|c|}{ Number of PN's } \\
Problem & $p=3$ & $p=5$ & $p=9$ & $p=17$ \\
\hline QP24 & 0.5645 & 0.5383 & - & - \\
QP25 & 0.7990 & 0.8853 & 0.7625 & - \\
QP26 & 1.381 & 1.465 & 1.299 & 0.6777 \\
\hline
\end{tabular}

† For each problem, the number $L$ of worker nodes varies from 2 to $Q+1$. Note that $p$ equals $L+1$, because one master node is used besides $L$ worker nodes. 
gradient computations per major iteration significantly vary from one processor to another. De Leone and Mangasarian [6] have presented an asynchronous parallel SOR method for the linear complementarity problem. It is one of the interesting future topics to develop an asynchronous version of the algorithm proposed in this paper.

\section{Acknowledgments}

The authors are grateful to one of the referees for very helpful comments and suggestions. They also thank Mr. Tsukasa Kimezawa of ATR Human Information Processing Research Laboratories for valuable discussions on the implementation strategy and the computer programs on the CM-5. This work was supported in part by the Scientific Research Grant-in-Aid from the Ministry of Education, Science and Culture, Japan (No. 06650443).

\section{References}

[1] Bertsekas, D.P. and Tsitsiklis, J.N.: Parallel and Distributed Computation: Numerical Methods. Prentice-Hall, Englewood Cliffs, New Jersey, 1989.

[2] Censor, Y.: Parallel Application of Block-Iterative Methods in Medical Imaging and Radiation Therapy. Mathematical Programming, Vol. 42 (1988), 307-325.

[3] Chen, R.J. and Meyer, R.R.: Parallel Optimization for Traffic Assignment. Mathematical Programming, Vol. 42 (1988), 327-345.

[4] Chen, G. and Teboulle, M.: A Proximal-Based Decomposition Method for Convex Minimization Problems. Mathematical Programming, Vol. 64 (1994), 81-101.

[5] Cottle, R.W., Pang, J.-S. and Stone, R.E.: The Linear Complementarity Problem. Academic Press, San Diego, California, 1992.

[6] De Leone, R. and Mangasarian, O.L.: Asynchronous Parallel Successive Overrelaxation for the Symmetric Linear Complementarity Problem. Mathematical Programming, Vol. 42 (1988), 347-361.

[7] Eckstein, J.: The Alternating Step Method for Monotropic Programming on the Connection Machine CM-2. ORSA Journal on Computing, Vol. 5 (1993), 84-96.

[8] Eckstein, J. and Bertsekas, D.P.: On the Douglas-Rachford Splitting Method and the Proximal Point Algorithm for Maximal Monotone Operators. Mathematical Programming, Vol. 55 (1992), 293-318.

[9] Eckstein, J. and Fukushima, M.: Some Reformulations and Applications of the Alternating Direction Method of Multipliers. Large Scale Optimization: State of the Art (eds. W.W. Hager, D.W. Hearn and P.M. Pardalos). Kluwer Academic Publishers, Dordrecht, The Netherlands, 1994, 115-134.

[10] Ferris, M.C.: Parallel Constraint Distribution in Convex Quadratic Programming. Mathematics of Operations Research, Vol. 19 (1994). 645-658.

[11] Frommer, A. and Mayer, G.: On the Theory and Practice of Multisplitting Methods in Parallel Computation. Computing, Vol. 49 (1992), 63-74.

[12] Fukushima, M.: Application of the Alternating Direction Method of Multipliers to Separable Convex Programming Problems. Computational Optimization and Applications, Vol. 1 (1992), 93-111.

[13] Fukushima, M.: The Primal Douglas-Rachford Splitting Algorithm for a Class of Monotone Mappings with Application to the Traffic Equilibrium Problem. Mathematical Programming, Vol. 72 (1996), 1-15.

[14] Fukushima, M., Haddou, M., Nguyen, V.H., Strodiot, J.-J., Sugimoto, T. and Yamakawa, E.: A Parallel Descent Algorithm for Convex Programming. Computational Optimization and Applications, Vol. 5 (1996), 5-37. 
[15] Golub, G. and Ortega, J.M.: Scientific Computing: An Introduction with Parallel Computing. Academic Press, San Diego, California, 1993.

[16] Hestenes, M.R.: Conjugate Direction Methods in Optimization. Springer-Verlag, New York, 1980.

[17] Iusem, A.N.: On the Convergence of Iterative Methods for Symmetric Linear Complementarity Problems. Mathematical Programming, Vol. 59 (1993), 33-48.

[18] Luo, Z.-Q. and Tseng, P.: On the Convergence of a Matrix Splitting Algorithm for the Symmetric Monotone Linear Complementarity Problem. SIAM Journal on Control and Optimization, Vol. 29 (1991), 1037-1060.

[19] Luo, Z.-Q. and Tseng, P.: Error Bound and Convergence Analysis of Matrix Splitting Algorithms for the Affine Variational Inequality Problem. SIAM Journal on Optimization, Vol. 2 (1992), 43-54.

[20] Machida, N., Fukushima, M. and Ibaraki, T.: A Multisplitting Method for Symmetric Complementarity Problems. Journal of Computational and Applied Mathematics, Vol. 62 (1995), 217-227.

[21] Mangasarian, O.L.: Nonlinear Programming. McGraw-Hill, New York, 1969.

[22] Mangasarian, O.L. and De Leone, R.: Parallel Successive Overrelaxation Methods for Symmetric Linear Complementarity Problems and Linear Programs. Journal of the Optimization Theory and Applications, Vol. 54 (1987), 437-446.

[23] Mangasarian, O.L. and De Leone, R.: Parallel Gradient Projection Successive Overrelaxation for Symmetric Linear Complementarity Problems. Annals of Operations Research, Vol. 14 (1988), 41-59.

[24] Nielsen, S.S. and Zenios, S.A.: Proximal Minimizations with D-Functions and the Massively Parallel Solution of Linear Network Programs. Computational Optimization and Applications, Vol. 1 (1993), 375-398.

[25] Thinking Machines Corporation: CM Fortran Programming Guide, Version 2.1. Cambridge, Massachusetts, 1994.

[26] Thinking Machines Corporation: CM-5 CM Fortran Performance Guide, Version 2.1. Cambridge, Massachusetts, 1994.

[27] Thinking Machines Corporation: CMMD Reference Manual, Version 3.0. Cambridge, Massachusetts, 1993.

[28] Zenios, S.A.: Data Parallel Computing for Network-Structured Optimization Problems. Computational Optimization and Applications, Vol. 3 (1994), 199-242.

[29] Zenios, S.A. and Censor, Y.: Massively Parallel Row-Action Algorithms for Some Nonlinear Transportation Problems. SIAM Journal on Optimization, Vol. 1 (1991), 373-400.

Eiki YAMAKAWA :

College of Business Administration,

Takamatsu University,

Takamatsu, Kagawa 761-01, Japan.

E-mail: yamakawa@takamatsu-u.ac.jp 